\title{
Performane Analysis of IEEE 802.11 WLAN by Varying PCF, DCF and EDCF to Enhance Quality of Service
}

\author{
Satyajit Sarmah \\ Department of IT \\ Gauhati University
}

\author{
S.K. Sarma \\ Department of IT \\ Gauhati University
}

\begin{abstract}
With the improvement of wireless network, quality of service has become a major research area. Researchers have proposed many co-ordination functions for improvement of service quality. In our study, we have compared the different characteristics and properties of PCF, DCF and Enhanced DCF to evaluate the performance of wireless network. We used OPNET IT GURU Academic Edition simulator for perform simulations. Protocols are tested under the realistic conditions to perform evaluation of the coordination functions. Various parameters such as Delay, Data Dropped, Throughput, Load, Retransmission Attempts Traffic Sent and Traffic Received in wireless network are tested to study the quality in various environments using OPNET network simulator. Different parameters are considered to study the impact of various co-ordination functions for wireless networks. In this paper we have studied the results obtained, and recommend the best possible protocol that can provide high QoS under respective network traffic conditions.
\end{abstract}

\section{Keywords}

PCF, DCF, EDCF, Data Dropped, Throughput, Traffic Sent, Traffic Received, Load, Retransmission Attempts.

\section{INTRODUCTION}

These wireless technologies are playing an increasingly prominent role in the entire Internet infrastructure. IEEE 802.11 is most widely and rapidly used standard now days for its simplicity and robustness. As wireless medium is a shared medium, so as more and more devices demand the bandwidth, performance has become a crucial issue of concern. So, apart from speed it will also have to assure the Quality of Service, especially when dealing with voice, video and other real time services [1]. IEEE 802.11 standard has two sub layers MAC protocols: Point Coordination Function (PCF) and Distributed Coordination Function (DCF). Point coordinators reside in access points, so the PCF is restricted to infrastructure networks [2][3]. To gain priority over standard contention based services, after a shorter interval PCF allows stations to transmit. Contention-free service is not provided full-time. Periods of contention-free service arbitrated by the point coordinator alternate with the standard DCF-based service [4].

When the PCF is used, time on the medium is divided into the contention-free period (CFP) and the contention period. Access to the medium in the former case is controlled by the PCF, while access to the medium in the latter case is controlled by the DCF. The DCF is the basis of the standard CSMA/CA access mechanism. Stations use a random backoff to avoid collisions after each frame, with the first transmitter seizing the channel. In some circumstances, the DCF may use the request-to-send/clear-to-send (RTS/CTS) clearing technique to further reduce the possibility of collisions. DCF is widely used in traffic, which provides a standard Ethernetlike contention-based service. DCF allows multiple independent stations to interact without central control, and thus may be used in either IBSS networks or in infrastructure networks [5]. A period called the contention window or backoff window follows the DIFS. This window is divided into slots. Stations pick a random slot and wait for that slot before attempting to access the medium. When several stations are attempting to transmit, the station that picks the first slot (the station with the lowest random number) wins.

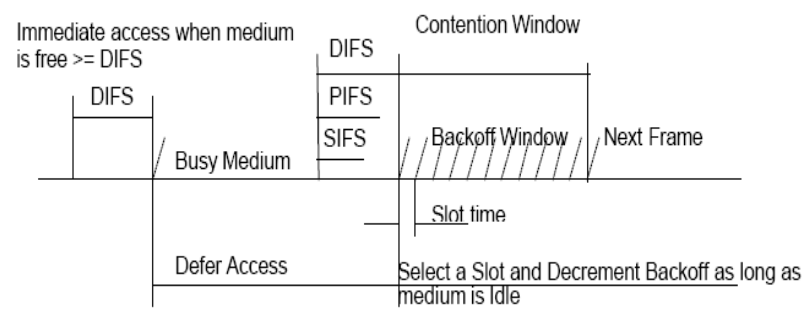

Fig. 1. Diagrammatic representation of DCF access method

With the improvement of wireless technology, a new component of 802.11 MAC called EDCF is introduced, which is the enhanced version of the legacy DCF. EDCF provides need based channel access to frames of different priorities as labeled by the higher layer. EDCF is a contention-based channel access scheme [6]. Total 8 delivery priorities are in EDCF for distributed access and differentiated service. Therefore, EDCF access channel uses at most eight prioritized output queues, one for each delivery priority, called Traffic Categories (TCs). The CWmin and CWmax parameters can be set differently for different traffic categories, such as, a high priority TC with small values of CWmin and CWmax and a low priority TC with higher values of CWmin and CWmax. Instead of using a DIFS, as a minimum specified idle duration time as defined in DCF, a new kind of interframe space called Arbitration Interframe Space (AIFS) is used [7].

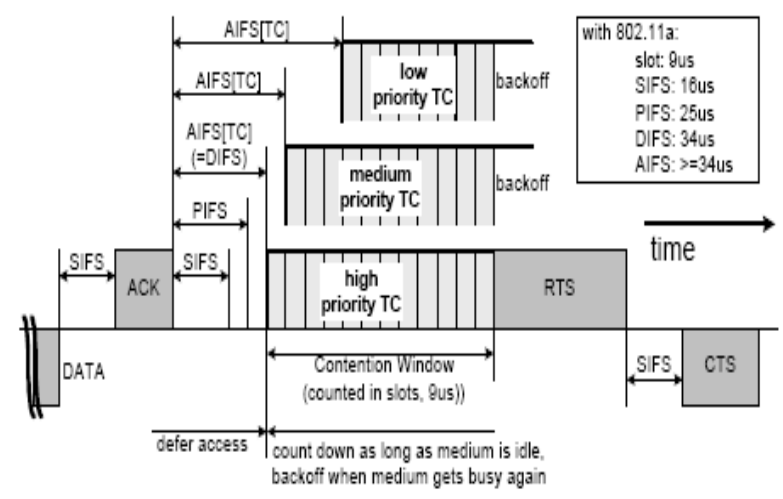

Fig.. 2. Diagrammatic representation of EDCF access method. 
Each frame from the higher layer arrives at the MAC along with a specific priority value in the MAC frame header.

\section{Table 1Access Priority}

\begin{tabular}{|c|c|c|}
\hline \hline Priority & $\begin{array}{c}\text { Access } \\
\text { Category(AC) }\end{array}$ & Designation \\
\hline 1 & 0 & Background \\
\hline 2 & 0 & Standard \\
\hline 0 & 1 & Best Effort \\
\hline 3 & 1 & Excellent Effort \\
\hline 4 & 2 & Interactive \\
\hline 5 & 2 & Interactive Voice \\
\hline 6 & 3 & \\
\hline 7 & 3 & \\
\hline & & \\
\hline
\end{tabular}

A single station may implement up to eight transmission queues realized as virtual stations inside a station, with QoS parameters that determine their priorities. If the counters of two or more parallel TCs in a single station reach zero at the same time, a scheduler inside the station avoids the virtual collision.

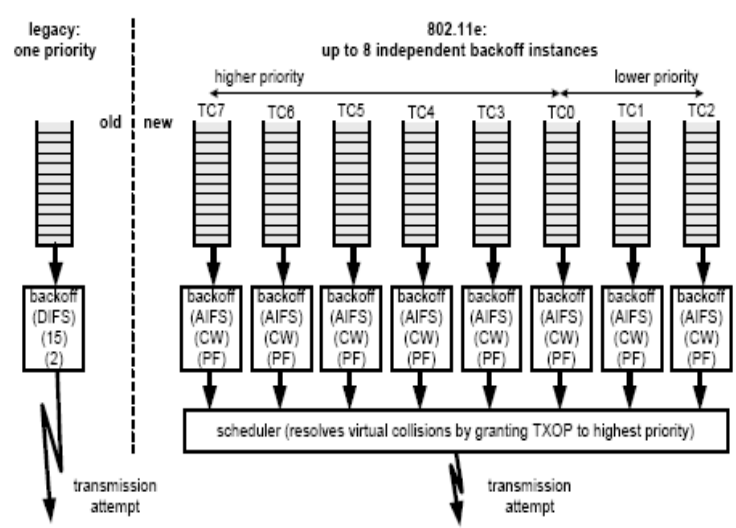

Fig. 3. Virtual back-off of eight traffic categories

After the introduction, a brief introduction to OPNET Simulator is given on second section. Our experimental scenarios and settings are discussed in third section. In fourth section, we have analyzed the results and finally in section five, conclude the paper.

\section{OPNET SIMULATOR}

OPNET (Optimized Network Engineering Tool) is a simulation tool for analyzing communication networks. The model description is done hierarchically. The user of OPNET graphically specifies the topology of his network which consists of nodes and links. Each node includes processors, queues, and traffic generators. The user also has to describe the data flow between components in a node. Finally, the behavior of each process is described using state diagrams. OPNET provides a comprehensive development environment for the specification, simulation and performance analysis of communication networks [8].

\section{EXPERIMENTAL SCENARIOS AND SETTINGS}

\subsection{Scenarios}

Our scenario has eighteen wireless LAN-based workstations in a simple network configuration (Infrastructured BSS) which demonstrates the PCF access method used by the Wireless LAN. PCF provides a contention-free $(\mathrm{CF})$ frame transfer. The medium access during the $\mathrm{CF}$ is regulated by the Point Coordinator (PC) which resides in the access point
(AP). The traffic flows between the stations have been configured as,

$$
\begin{aligned}
& \text { PCF_wkstn } 1 \text { <-------> PCF_wkstn } 2 \\
& \text { PCF_wkstn } 3 \text { <--------> PCF_wkstn } 4 \\
& \text { PCF_wkstn } 5 \text { <--------> PCF_wkstn } 6 \\
& \text { PCF_wkstn } 7 \text { <--------> PCF_wkstn } 8 \\
& \text { PCF_wkstn } 9 \text { <-------> PCF_wkstn } 10 \\
& \text { PCF_wkstn } 11 \text { <-------> PCF_wkstn } 12 \\
& \text { PCF_wkstn } 13 \text { <-------> PCF_wkstn } 14 \\
& \text { PCF_wkstn } 15 \text { <--------> PCF_wkstn } 16 \\
& \text { PCF_wkstn } 17 \text { <--------> PCF_wkstn } 18
\end{aligned}
$$

All the PCF related configuration parameters are grouped into a single compound attribute "PCF Parameters". The stations can participate in frame transfers during $\mathrm{CF}$ period by enabling the "PCF Functionality" attribute on the work stations.

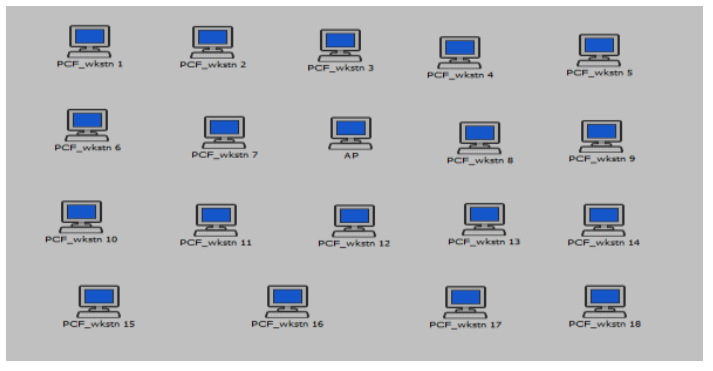

Fig. 4. OPNET simulation setup for PCF

The second scenario has eighteen wireless LAN-based workstations in a simple network configuration (Infrastructured BSS) which demonstrates the DCF access method used by the Wireless LAN. DCF provides a contention-Period (CP) frame transfer. The traffic flows between the stations have been configured as,

$$
\begin{aligned}
& \text { DCF_wkstn } 1<------->\text { DCF_wkstn } 2 \\
& \text { DCF_wkstn } 3<------->>\text { DCF_wkstn } 4 \\
& \text { DCF_wkstn } 5 \text { <-------> DCF_wkstn } 6 \\
& \text { DCF_wkstn } 7 \text { <-------> DCF_wkstn } 8 \\
& \text { DCF_wkstn } 9 \text { <-------> DCF_wkstn } 10 \\
& \text { DCF_wkstn } 11<------->\text { DCF_wkstn } 12 \\
& \text { DCF_wkstn } 13<------->\text { DCF_wkstn } 14 \\
& \text { DCF_wkstn } 15<------>\text { DCF_wkstn } 16 \\
& \text { DCF_wkstn } 17 \text { <-------> DCF_wkstn } 18
\end{aligned}
$$

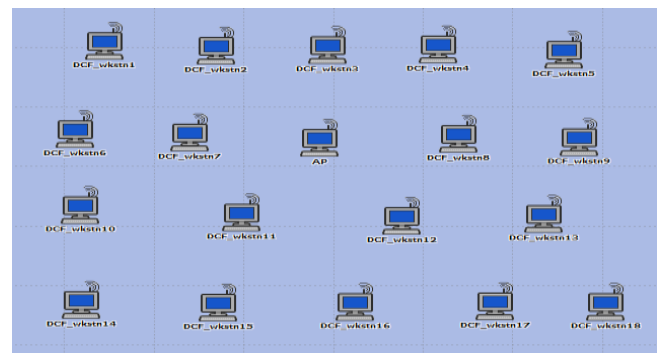

Fig. 5. OPNET simulation setup for DCF 
This scenario has eighteen wireless LAN-based workstations in a simple network configuration (Infrastructured BSS) which demonstrates the EDCF access method used by the Wireless LAN. The traffic flows between the stations have been configured as,
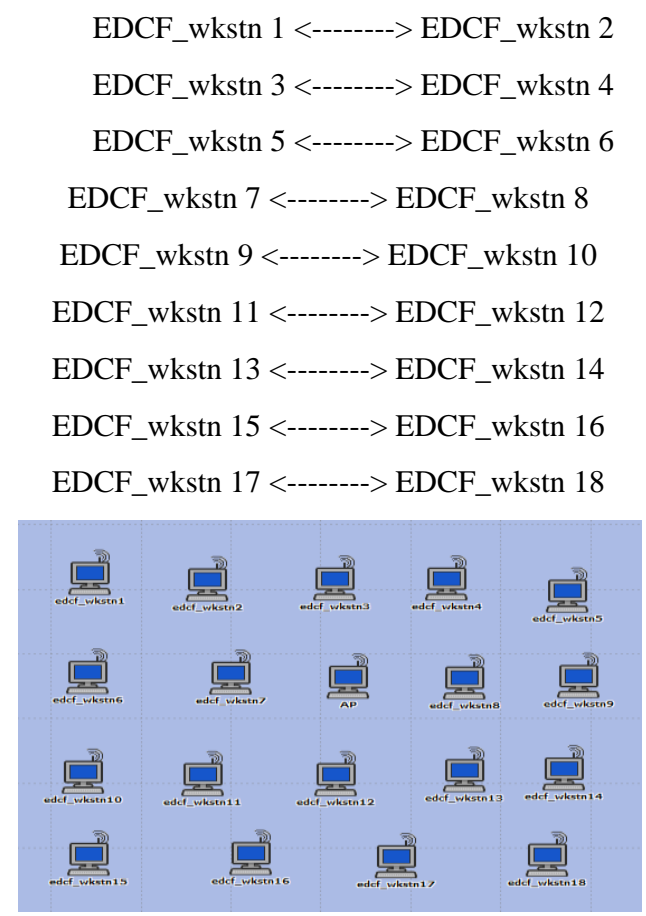

Fig. 6. OPNET simulation setup for EDCF

\subsection{Simulation Parameter}

\begin{tabular}{|c|l|c|}
\hline Application & \multicolumn{1}{|c|}{ Parameters } & Units \\
\hline \multirow{4}{*}{ WLAN } & Throughput & Bits/Sec \\
\cline { 2 - 3 } & Data Traffic Received & Bits/Sec \\
\cline { 2 - 3 } & Data Traffic Sent & Bits/Sec \\
\cline { 2 - 3 } & Dropped Data Packet & Bits/Sec \\
\cline { 2 - 3 } & Retransmission Attempts & Packets \\
\cline { 2 - 3 } & & Bits/Sec \\
\cline { 2 - 3 } & Load & \\
\hline
\end{tabular}

\subsection{Traffic Generation Parameters}

\begin{tabular}{cl}
\hline Traffic Generation Parameters & $(\ldots)$ \\
\hline . Start Time (seconds) & constant (0.02) \\
ON State Time (seconds) & exponential (10) \\
OFF State Time (seconds) & exponential (90) \\
\hline . Packet Generation Arguments & $(\ldots)$ \\
\hline Interarival Time (seconds) & exponential (1.0) \\
. Packet Size (bytes) & exponential (1024) \\
\hline .. Segmentation Size (bytes) & No Segmentation \\
\hline Stop Time (seconds) & Never
\end{tabular}

Fig. 7. Traffic Generation Parameters

\subsection{WLAN Parameters \\ G Wireless LAN \\ -Wireless LAN MAC Address \\ $\because$ Wireless LAN Parameters \\ . BSS Identifier \\ Access Point Functionality Physical Characteristics \\ - Data Rate (bps) \\ $\oplus$ Channel Settings \\ Rits Threshold (bytes) \\ Fragmentation Threshold (bytes) - \\ CTSto-self Option \\ Short Retry Limit \\ AP Beacon Interval (secs) \\ Max Receive Lifetime (secs) \\ Buffer Size (bits) \\ Roaming Capability \\ Large Packet Processin \\ Auto Assigned \\ Enabled \\ irect Sequence \\ 11 Mbps \\ Enabled \\ 7 \\ 4 \\ 0.5 \\ 1024000 \\ 1024000 Drop}

Fig. 8. Different WLAN Parameters

\section{RESULT ANALYSIS}

We have simulated our models and six plotted graphs on six different parameters are selected for analysis. Graphs are combination of PCF, DCF and EDCF scenarios. Graphs are described below

\subsection{Traffic Received}

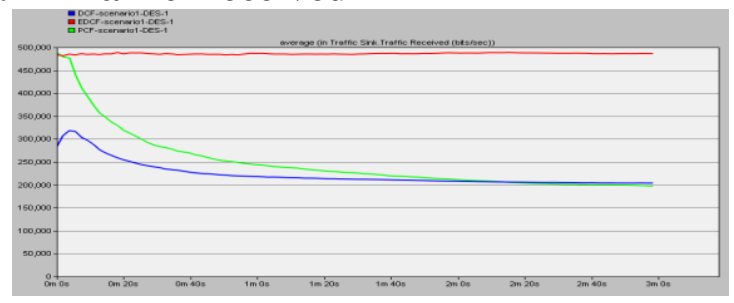

Fig. 9. Average Wireless LAN Traffic Received

It has been clearly observed that traffic data sent value of EDCF is better than PCF \& DCF.

\subsection{Traffic sent}

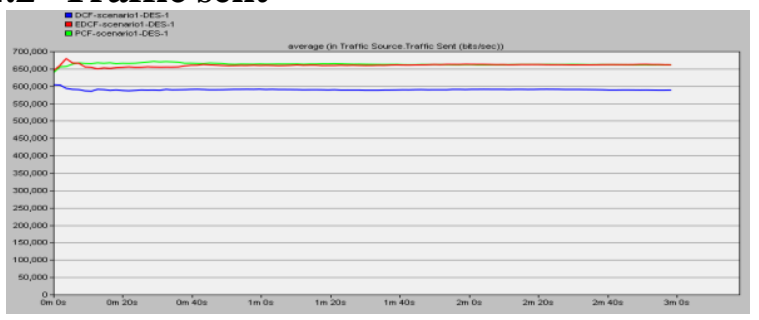

Fig. 10. Average Wireless LAN Traffic Sent

It has been noticed that for the first 20 seconds EDCF varies better than PCF and DCF. After that EDCF graph has merged with PCF graph. So it is concluded that the results are better with EDCF.

\subsection{Data Dropped}

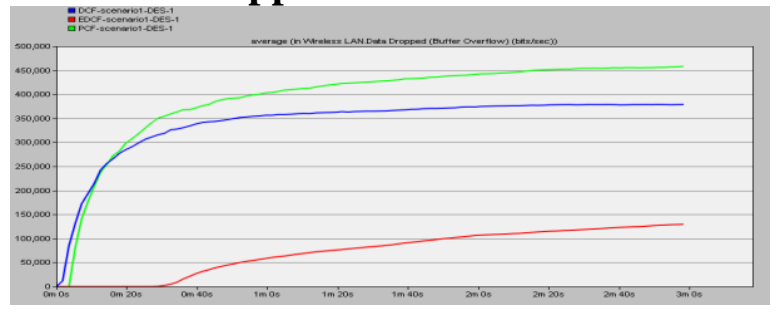

Fig. 11. Average Wireless LAN Data Dropped 
It has been noticed that for the first 30 seconds there is no data dropped for EDCF. After that EDCF has comparatively minimal data dropped than PCF and DCF. Therefore results are better with EDCF.

\subsection{Load}

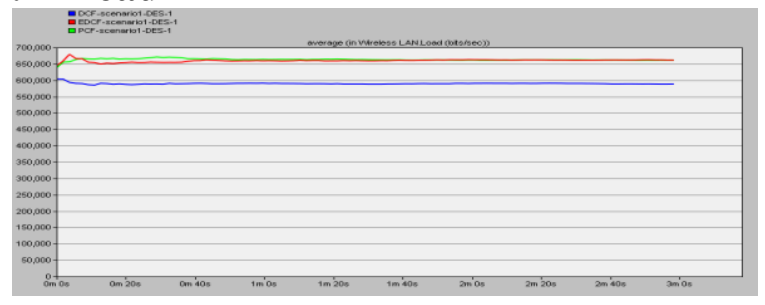

Fig. 12. Average Wireless LAN Load

\subsection{Retransmission Attempts}

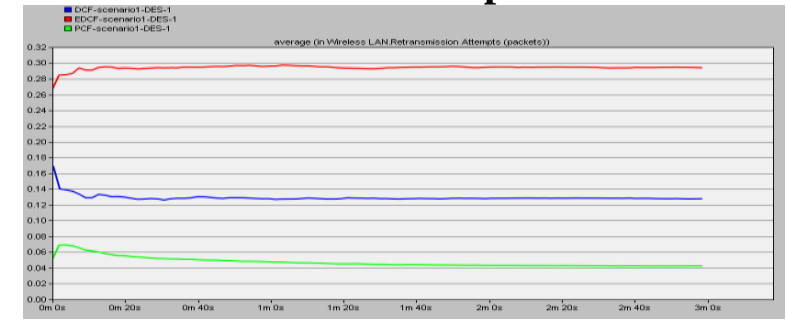

Fig. 13. Average Wireless LAN Retransmission Attempts

It has been noticed that Retransmission Attempts packets per second for EDCF is fairly more than PCF and DCF. Moreover, PCF has the lowest attempts of retransmission packets

\subsection{Throughput}

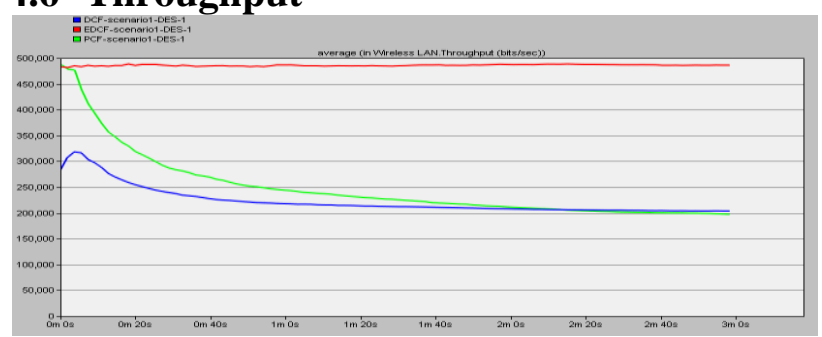

Fig. 14. Average Wireless LAN Throughput

It has been clearly noticed that for the first one minute throughput graph for both PCF and DCF decreases. At the same time EDCF graph shows a constant throughput during simulation. Therefore it is concluded that the results are better with EDCF.

\section{CONCLUSION}

In our study, we have checked the performance of wireless network for different parameters like Data Traffic Received,
Data Traffic Sent, Data Dropped, Load, Retransmission attempts and throughput using DCF, PCF and EDCF coordination functions. As a result of our study we have analyzed that network having EDCF coordination function is useful to improve quality of service. PCF \& DCF are basic access mechanism in WLAN which do not offer service differentiation to different types of traffic. So higher priority traffic like video streaming and voice applications suffer with large delay. EDCF on the other hand offers service differentiation on the basis of priorities and provide performance improvement over DCF mode. The performance of EDCF can further be improved by proper tunings of various parameters like physical characteristics, transmitted power, Fragmentation Threshold etc. So at the end of our study we can conclude that wireless network having EDCF co-ordination function can be used for getting better Quality of Service.

\section{ACKNOWLEDGMENT}

We are grateful to all faculty and staff members of Dept of IT, Gauhati University, for their valued suggestions and inputs during the work.

\section{REFERENCES}

[1] Qutaiba I. Ali, Performance Evaluation of WLAN Internet Sharing Using DCF PCF Modes.

[2] Sobrinho Joao L., Krishnakumar A. S., "Quality-ofService in Ad Hoc Carrier Sense Multiple Access Wireless Networks", IEEE journal on selected areas in communications, vol. 17, no. 8, august 1999.

[3] Rajaraman Rajmohan, "Wireless Networks", Pearson Edu,2005.

[4] Inderjeet Kaur, Manju Bala , Harpreet Bajaj, Performance Evaluation of Wlan by Varying Pcf, Dcf and Enhanced Dcf Slots To Improve Quality of Service, IOSR Journal of Computer Engineering (IOSRJCE), ISSN: 2278-0661 Volume 2, Issue 5 (July-Aug. 2012), PP 29-33.

[5] Rahul, V. and Dr. R. K. Bansal., "Simulation \& performance analysis of wired and wireless computer networks", International Journal of Computer Applications, February 2011.

[6] Rahul, V. and Dr. R. K. Bansal., "Simulation \& performance analysis of wired and wireless computer networks", International Journal of Computer Applications, February 2011.

[7] Rahul, V. and Dr. R. K. Bansal., "Simulation \& performance analysis of wired and wireless computer networks", International Journal of Computer Applications, February 2011.

[8] OPNET LABS, "Creating Wireless Network”, 2006. 